\title{
Comparison of two sperm processing techniques for ICSI: sperm swim-up and discontinuous density gradient centrifugation
}

\author{
Ruiz Nerea; Escalante Elisa; Moreno Pilar; Palomo Maria Luisa; de la Fuente Laura. \\ Human Reproduction Unit Obstetrics \& Gynaecology. Hospital 12 de Octubre. Madrid. Spain.
}

Study question: Does discontinuous density gradient centrifugation sperm preparation improve ICSI outcomes when compared to swim-up?

Summary answer: No advantage could be found for discontinuous density gradient centrifugation respect swim-up in terms of fertilization, pregnancy and implantation rate when ICSI technique was performed.

What is known already: Usually sperm capacitation in assisted human reproduction is performed in IVF laboratories using two specific techniques trying to imitate the in-vivo female genital track capacitation. The method is selected according to the sperm quality sample and the final ART procedure. The effectiveness of one sperm preparation procedure over another is still a controversial as many different procedures have been compared in heterogeneous conditions. The elected technique will be selected considering results laboratory management implications.

Study design, size, duration: This is a retrospective study, of 357 semen samples capacitated for ICSI procedure at the Human Reproduction Unit of Hospital Universitario 12 de Octubre, Madrid, Spain, in a public IVF programme in 2015-2016 period.

Participants/materials, setting, methods: The capacitation technique divided our study in two groups indistinctly, 170 samples were processed using swim-up and 187 with discontinuous density gradient (Nidacon Internacional, Flöjelbergsgatan 16 B 43137 Möindal, Sweden). Seminal parameters were analyzed before and after processing.

Main results and the role of chance: Improvements of the concentration of motile sperm were observed with both used techniques. Discontinuous density gradient centrifugation improved concentration recovery rate reaching statistically higher levels than swim-up procedure $\left(13,51 \times 10^{6} / \mathrm{ml}\right.$ vs $\left.8,16 \times 10^{6} / \mathrm{ml}\right)(p \leq 0,01)$. This marked difference respecting the concentration sperm recovery, doesn't affect other cycle variables analyzed: fertilization rate $(61,88 \%$ vs $59,75 \%)$, number of embryos per patient (2,36 vs 2,36$)$, good quality embryos per patient $(0,94$ vs 0,85$)(p>0,01)$. Neither pregnancy rate nor implantation rate per transfers were affected by the sperm processing technique $(30,00 \%$ vs $26,27 \% ; 22,92 \%$ vs $17,64 \%$ respectively) ( $p>0,01)$.

According to our results the discontinuous density gradients do not improve ICSI outcomes even though a significant increase in concentration of motile sperm rates made evident. In conclusion, using discontinuous density gradient is not justified in semen samples for ICSI even though ART in which higher post-processing concentration of motile sperm rates is required could benefit of this procedure like IUI.
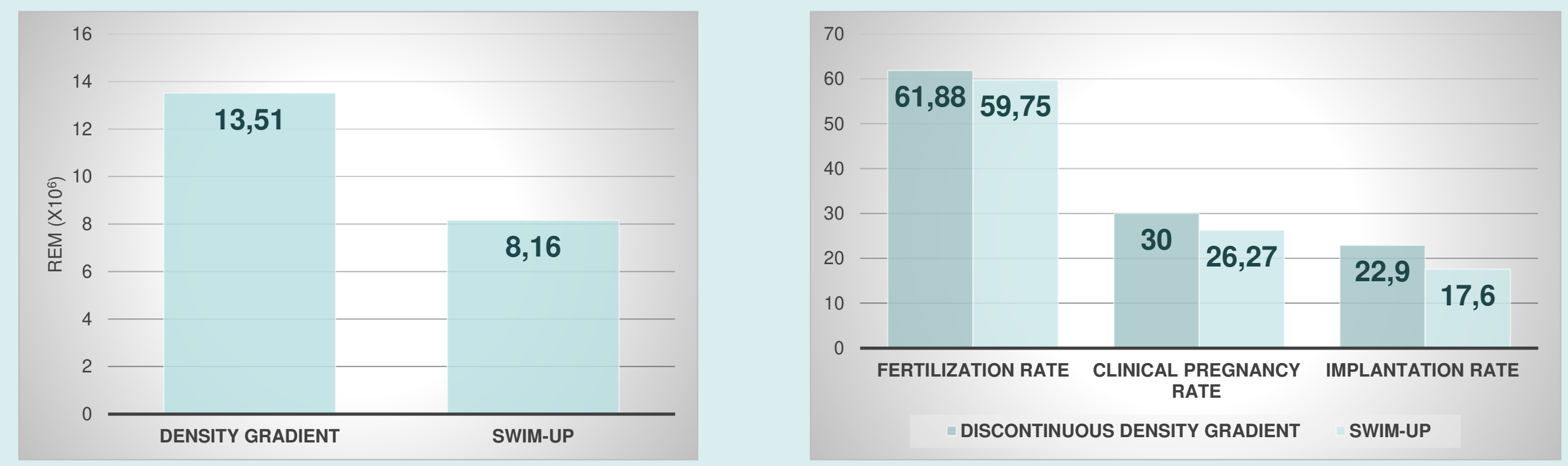

Conclusions: When only a few spermatozoa need to be selected, as it is the case in ICSI technique, sperm selection with discontinuous density gradients has no effect in cycle outcome parameters. Election of sperm processing technique for ICSI should be based on other kind of parameters: availability, costs, laboratory organization, etc. 